\title{
Predicting a company's performance
}

\author{
G. Kurniawan \& R. Rokhim \\ Faculty of Economics and Business, Universitas Indonesia, Indonesia
}

\begin{abstract}
This study analyzed whether the elbow method and K-means clustering can help investors diversify their investment in different companies. Using clustering in machine learning and big data, a method was generated to categorize a list of companies with similar performances based on their fundamental analysis. Companies' fundamental analysis variables were used to classify stock data using K-means clustering application. In the end, companies with similar stock performances will be grouped, and it will be easier for investors to diversify their investments.
\end{abstract}

\section{INTRODUCTION}

There are various considerations investors will have when they are investing in company's stock. With investors' consideration and the risk each of investments has, investing will undoubtedly be more complex and varied. The risk in the stock portfolio itself will vary according to the proportions held by individuals or institutions (Statman, 1987).

According to Statman (1987), the risk of investing in a company's stock can be minimized by choosing different types or categories of stocks. The act of selecting different stocks in a stock portfolio is often known as stock diversification. The risks arising from investments can be minimized by carrying out portfolio selection activities. Portfolio selection activity is an activity to select stocks for investment or diversification. Clustering is an efficient method of selecting stocks for portfolio management. This method is also considered to be able to minimize the risks involved in determining stocks when carrying out portfolio management. One of the clustering methods commonly used is K-means clustering (Ren et al., 2017).

This study focuses on how K-means clustering can be used to diversify stocks to minimize the risk that is inherent in each investment. Five fundamental analysis variables were used to classify stock data from Indonesia (Kompas100). This study also seeks to form a K-means clustering algorithm that can reduce the risk of investing in stocks using the diversification method.

\section{LITERATURE REVIEW}

Clustering is commonly used to group similar stocks in one group. The clustering method for the stock market has been done in Australia (Aitken et al., 1996), China (Liao \& Chou, 2013), and India (Nanda et al., 2010). It is expected to assist in determining investment in the stock market. One of the clustering methods commonly used in machine learning is K-means clustering.

$\mathrm{K}$-means clustering aims at looking for similarities between data and grouping the similar data into a cluster or a group. According to Bishop (2014), K-means clustering divides a data set composed of $\mathrm{N}$ observations into $\mathrm{K}$ clusters. The distribution of data to each cluster in this method is done by considering the smallest distance between the data and the average of each cluster. The determination of cluster values to be carried out using the K-means clustering method is usually determined subjectively. 
However, to be more certain, one method is used to ensure that the selection of cluster values is correct. This method is the elbow method. The elbow method has been used by Bholowalia and Kumar (2014) in determining the right number of clusters in the K-means clustering algorithm.

This method is used to observe a graph depicting a cluster value whose sum square of errors drop to a sudden lowest point.

\section{METHODOLOGY}

\subsection{Research data}

The data used in this study were retrieved from stock data for public companies traded in the Indonesia Stock Exchange (IDX) and from public companies listed in the Kompas100 index. Five companies' financial ratios will be used as a variable to perform the clustering analysis.

\subsection{Research model}

The procedures of this research are as follows:

1. Retrieving stock data from websites commonly used for stocks news;

2. Applying elbow method to determine the correct number of clusters;

3. Inputting the stock data into the K-means clustering algorithm;

4. Analyzing the results of the grouping of stock data into each cluster; and

5. Determining the possible investment strategies that can be chosen by investors for each cluster.

\subsection{Elbow method}

The elbow method was commonly used by analysts to determine the right number of clusters to be included in the clustering algorithm. It can also be applied to the data by determining the sum square of errors in each variable. The sum square of errors for each variable can be calculated by entering the cluster values with iteration (Bholowalia \& Kumar, 2014). The following model is the elbow method objective function:

$$
S S E=\sum_{K=1}^{k} \sum_{x i \in S_{k}}\left\|X_{i}-C_{k}\right\|_{2}^{2}
$$

\section{$3.4 \quad$ K-means clustering}

The clustering method is used to group similar stocks into one cluster. K-means clustering used the smallest distance analysis with its average in classifying similar data. This smallest distance is calculated using the Euclidean distance that is widely used in the K-means clustering algorithm (Bishop, 2014). The following model is the K-means clustering objective function:

$$
\begin{aligned}
& J=\sum_{n=1}^{N} \sum_{k=1}^{K} r_{n k}\left\|x_{n}-\mu_{k}\right\|^{2} \\
& \text { With, } r_{n k}=\left\{1, \text { if } k=\operatorname{argmin}_{j}\left\|x_{n}-\mu_{j}\right\|^{2}, x_{n}=n \text { Data, } \mu_{k}=\text { Mean } k 0,\right. \text { others }
\end{aligned}
$$

The results of this cluster can be used as a reference for providing recommendations to investors in making an investment in certain stocks. It can also be used by investors and other institutions in finding the right investment and diversifying stocks to get the expected profit (Nanda et al., 2010). 


\section{RESULTS}

\subsection{Elbow method results}

The elbow method was used to determine the right number of clusters, which will be used for $\mathrm{K}$-means clustering. There are six clusters that were determined by the elbow method.

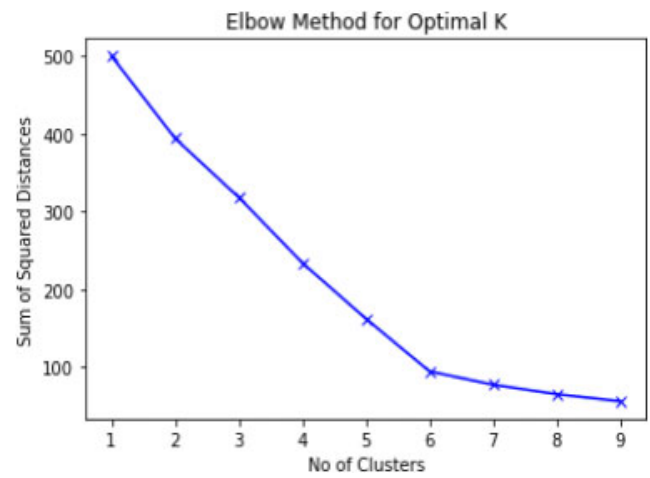

Figure 1. Elbow method.

According to the elbow method, the best cluster to be processed by the K-means clustering method is the sixth cluster.

\section{$4.2 \quad K$-means clustering results}

From the six clusters, there are several members of clusters that were achieved using K-means clustering.

Table 1. Number of cases in each cluster

Number of Cases in Each Cluster

\begin{tabular}{lr}
\hline Cluster 1 & 1 \\
Cluster 2 & 20 \\
Cluster 3 & 2 \\
Cluster 4 & 3 \\
Cluster 5 & 1 \\
Cluster 6 & 73 \\
\hline
\end{tabular}

Table 2. Final cluster centers.

\begin{tabular}{|c|c|c|c|c|c|c|}
\hline \multirow[b]{2}{*}{ Final Cluster Centers } & \multicolumn{6}{|l|}{ Clusters } \\
\hline & 1 & 2 & 3 & 4 & 5 & 6 \\
\hline PBV & 8.25287 & .52504 & -.19753 & .64530 & 1.72116 & -.30158 \\
\hline EPS & -.02046 & -.12925 & 6.04085 & -.32254 & -.98991 & -.10300 \\
\hline ROA & 4.69248 & 1.28890 & .99357 & -.48792 & -2.06914 & -.39623 \\
\hline ROE & 3.83807 & .53509 & .42988 & -.24060 & -8.27951 & -.08765 \\
\hline $\mathrm{P} /$ E Ratio & .18255 & -.00418 & -.14280 & 5.03817 & -.23212 & -.20131 \\
\hline
\end{tabular}


Each cluster from K-means clustering has different characteristics. This characteristic can be seen in the results of K-means clustering with the descriptive analysis on the data.

Cluster 1: High price to book value stock

Cluster 2: Moderate price to book value, ROA, and ROE stock

Cluster 3: High earnings per share stock

Cluster 4: High price to earnings ratio stock

Cluster 5: Low ROE and ROA stock

Cluster 6: Moderately low in all variables stock

According to the analysis, investors can get six clusters with various characteristics. They can do some investment diversification within the six clusters, in which the investment diversification should be based on investors' behavior. In this matter, investors could do investment in some ways that they believe is the strongest or better way to gain profit.

\section{CONCLUSIONS AND RECOMMENDATIONS}

\subsection{Conclusions}

K-means clustering can be used to cluster the same category of stocks based on company's performance that can be seen through company's fundamental analysis. According to the analysis, K-means clustering grouped company stocks' data into six clusters based on five fundamental ratios. Clusters that were built by K-means clustering can be used by investors to diversify their stock portfolio. Diversification within the stock portfolio can be a way to reduce stock investment risk.

\subsection{Recommendations}

The analysis that was done in this study was based on data from only hundred stocks, which means that the development of this study could be done in another field such as in different index or different stocks' data. Variables used in this study could also be enlarged by other variables suitable to the study. A lot of progress and development could be made with this study. Another conclusion and clustering could also be found if there are different variables and data.

\section{REFERENCES}

Aitken, M., Brown, P., Buckland, C., Izan, H. Y., \& Walter, T. (1996). Price clustering on the Australian stock exchange. Pacific Basin Finance Journal. https://doi.org/10.1016/0927-538x(96)00016-9

Bholowalia, P., \& Kumar, A. (2014). EBK-Means: A Clustering Technique based on Elbow Method and K-Means in WSN. In International Journal of Computer Applications.

Bishop, C. M. (2014). Bishop - Pattern Recognition And Machine Learning - Springer 2006. Antimicrobial Agents and Chemotherapy. https://doi.org/10.1128/AAC.03728-14

Liao, S. H., \& Chou, S. Y. (2013). Data mining investigation of co-movements on the Taiwan and China stock markets for future investment portfolio. Expert Systems with Applications. https://doi.org/ 10.1016/j.eswa.2012.08.075

Nanda, S. R., Mahanty, B., \& Tiwari, M. K. (2010). Clustering indian stock market data for portfolio management. Expert Systems with Applications. https://doi.org/10.1016/j.eswa.2010.06.026

Ren, F., Lu, Y. N., Li, S. P., Jiang, X. F., Zhong, L. X., \& Qiu, T. (2017). Dynamic portfolio strategy using clustering approach. PLoS ONE. https://doi.org/10.1371/journal.pone.0169299

Statman, M. (1987). How Many Stocks Make a Diversified Portfolio? The Journal of Financial and Quantitative Analysis. https://doi.org/10.2307/2330969 